\title{
A re-assessment of strong line metallicity conversions in the machine learning era.
}

\author{
Hossen Teimoorinia ${ }^{1,2}$, Mansoureh Jalilkhany ${ }^{3}$, Jillian M. Scudder ${ }^{4}$, Jaclyn Jensen ${ }^{2}$, \\ Sara L. Ellison ${ }^{2}$ \\ ${ }^{1}$ NRC Herzberg Astronomy and Astrophysics, 5071 West Saanich Road, Victoria, BC, V9E 2E7, Canada \\ ${ }^{2}$ Department of Physics and Astronomy, University of Victoria, Victoria, BC, V8P 5C2, Canada \\ ${ }^{3}$ Department of Computer Science, University of Victoria, Victoria, BC, V8W 2Y2, Canada \\ ${ }^{4}$ Department of Physics and Astronomy, Oberlin College, Oberlin, Ohio, OH 44074, USA
}

February 16, 2021

\begin{abstract}
Strong line metallicity calibrations are widely used to determine the gas phase metallicities of individual HII regions and entire galaxies. Over a decade ago, based on the Sloan Digital Sky Survey Data Release 4 (SDSS DR4), Kewley \& Ellison published the coefficients of third-order polynomials that can be used to convert between different strong line metallicity calibrations for global galaxy spectra. Here, we update the work of Kewley \& Ellison in three ways. First, by using a newer data release (DR7), we approximately double the number of galaxies used in polynomial fits, providing statistically improved polynomial coefficients. Second, we include in the calibration suite five additional metallicity diagnostics that have been proposed in the last decade and were not included by Kewley \& Ellison. Finally, we develop a new machine learning approach for converting between metallicity calibrations. The random forest algorithm is non-parametric and therefore more flexible than polynomial conversions, due to its ability to capture non-linear behaviour in the data. The random forest method yields the same accuracy as the (updated) polynomial conversions, but has the significant advantage that a single model can be applied over a wide range of metallicities, without the need to distinguish upper and lower branches in $R_{23}$ calibrations. The trained random forest is made publicly available for use in the community.
\end{abstract}

Key words: galaxies: abundances — galaxies: fundamental parameters - methods: data analysis - astronomical data bases: surveys - methods: statistical

\section{INTRODUCTION}

The gas phase metallicity of a galaxy is a fundamental quantity that captures the cumulative history of stellar chemical enrichment, outflows and delivery of pristine material from the intergalactic medium. Commonly denoted as $12+\log (\mathrm{O} / \mathrm{H})$, this metallicity is encoded into the emission line spectrum of an HII region or galaxy, and can be derived 'directly' by solving for the electron temperature and density. Electron densities are readily determined from nebular doublets, such as [OII] $\lambda \lambda 3726,3729$. In theory, electron temperatures $\left(T_{e}\right)$ are similarly readily obtained, by measuring the relative strengths of emission lines of a given species that originate from different upper energy levels, e.g. [OIII] $\lambda 5007$ and the auroral line [OIII] $\lambda 4363$.

However, in practice, the weakness of the auroral lines that are used to measure $T_{e}$ limits this method to metallicities below approximately $12+\log (\mathrm{O} / \mathrm{H}) \sim 8.3$ for most individual spectra. The auroral line becomes weaker as metallicity increases due to the increased cooling in the interstellar medium. A common alternative practice is therefore to use 'strong line methods' which use readily detectable nebular emission line ratios that have been calibrated against 'known' metallicities (see Kewley et al.2019 for a review). A large variety of strong line metallicity calibrations exists in the literature, broadly falling into two categories; the so-called 'empirical' methods, that are calibrated against direct $\left(T_{e}\right)$ metallicity measurements (e.g., Pettini \& Pagel 2004, Marino et al.|2013, Curti et al. 2017) and those that are calibrated against theoretical models (e.g., McGaugh 1991; Zaritsky et al.|1994, Kewley \& Dopita 2002)

Although the strong line metallicity calibrations show good internal consistency, there are strong systematic offsets between them. Therefore, attempting to combine published measurements of gas phase metallicity that have used different metallicity calibrations will result in error, with offsets as large as 0.7 dex (e.g., Kewley \& Ellison 2008). In order to facilitate the combination of metallicities derived using different methods, Kewley \& Ellison (2008) published an extensive assessment of the major strong line metallicity diagnostics of the time. Using metallicity data for $\sim 28,000$ 
galaxies in the Data Release 4 (DR4) of the Sloan Digital Sky Survey (SDSS), Kewley \& Ellison (2008) published tables of coefficients for third-order polynomials that could be used to convert between any pair of diagnostics used in their study.

Although the metallicity conversions of Kewley \& Ellison (2008) continue to be widely used, in the decade since their publication several advances have been made in the community. First, the samples of star-forming galaxies that can be used to calibrate metallicities has grown considerably. Secondly, several new metallicity calibrations have been introduced in the literature for which no conversions (to other diagnostics) exist. Finally, the rapid growth of machine learning technologies means that new methods exist for calibrating between different diagnostics. Such methods offer numerous advantages over the polynomial fitting approach of Kewley \& Ellison 2008). For example, they can capture non-linearities in the data, make no assumptions on the form of the conversion (viz the third-order polynomial used in Kewley \& Ellison 2008) and simplify the process by avoiding different functional forms in different regimes (e.g. upper and lower metallicity branches).

Machine learning has already gained a solid foothold in astronomical data processing. For example, neural networks and deep learning methods have been widely used for both image classification (Bottrell et al. 2019, Huertas-Company et al. 2019, Ćiprijanović et al. 2020, Ferreira et al. 2020, Teimoorinia et al. 2020a b), ranking tasks (Teimoorinia et al. 2016; Bluck et al. 2019, Dey et al. 2019, Ellison et al. 2020), as well as for regression and pattern recognition applications (Ellison et al. 2016, Teimoorinia et al. 2017). In the realm of interstellar medium studies, neural networks and other techniques, such as random forests, have also been used to predict emission line fluxes (Teimoorinia \& Ellison 2014) and metallicities from either broad band photometry (Acquaviva et al.2015) or spectra (Ucci et al. 2018; Ho 2019).

Given the advances made since the original work of Kewley \& Ellison (2008), the goal of the work presented here is three-fold. First, using a larger sample of star-forming galaxies drawn from the SDSS Data Release 7 (DR7; Sec 2), in Sec. 3 we re-assess the polynomial-based metallicity conversions presented by Kewley \& Ellison (2008). We show that there are small systematic errors (up to $\sim 0.1 \mathrm{dex}$ ) when the old Kewley \& Ellison (2008) conversions are applied to the newer DR7 sample. New coefficients are tabulated, providing a statistically improved set of functions for researchers desiring to use polynomial based conversions between metallicity diagnostics (Sec. 3.1. Second, we extend the original suite of metallicity diagnostics with a further five calibrations that have been presented in the literature since the original work of Kewley \& Ellison (2008). Finally, in Sec. 4 we develop a random forest alternative to the polynomial conversion approach. We emphasize that our goal is not to develop new metallicity calibrations, but rather to facilitate the conversion between existing methods using modern samples and methods. Our results are summarized in Sec. 5

\footnotetext{
${ }^{1}$ Despite its long presence in the literature, the DR7 remains one of the most widely used galaxy datasets for spectroscopic work in the nearby universe, thanks to the public MPA/JHU database of physical properties, including emission line fluxes.
}

\section{DATA}

\subsection{A brief review of Kewley \& Ellison (2008)}

We refer the reader to more extensive treatises on metallicity measurements in galaxies for a full pedagogical description and theoretical framework of this field (e.g., Pagel 1989, Osterbrock 1989, Stasińska 2006, López-Sánchez et al. 2012, Pérez-Montero \& Amorín 2017; Kewley et al. 2019). Here, we review only the essential concepts, data selection and metallicity calibrations used in Kewley \& Ellison (2008) as context for our re-assessment of that work.

Strong line metallicity diagnostics combine the fluxes from two or more nebular emission lines, which can be input into (usually, simple polynomial) functions that have been either empirically or theoretically calibrated in order to solve for the gas phase metallicity, $\mathrm{O} / \mathrm{H}$. The function that converts line fluxes to $\mathrm{O} / \mathrm{H}$ are not always monotonic. One of the most well known examples of a strong line diagnostic that is double valued in $\mathrm{O} / \mathrm{H}$ for a given line ratio, is the family of $R_{23}$ diagnostics, where

$R_{23}=\frac{[O I I] \lambda 3727+[O I I I] \lambda 4959+[O I I I] \lambda 5007}{H \beta}$

Additional constraints are required to break the $R_{23}$ degeneracy in order to determine whether the galaxy (or HII region) lies on the upper or lower branch of the calibration. Kewley \& Ellison (2008) use the ratio of [NII]/[OII] to break the $R_{23}$ degeneracy, since this ratio is not sensitive to ionization parameter and largely monotonic with metallicity in the range of SDSS galaxy metallicities. Whilst this approach can work well for metal-rich and metalpoor galaxies, at intermediate metallicities the calibration can be ambiguous and $\mathrm{O} / \mathrm{H}$ is more uncertain.

Kewley \& Ellison (2008) select their galaxy sample from the SDSS DR4. A S/N of at least 8 is required in the [OII], [OIII], $[\mathrm{NII}],[\mathrm{SII}]$ and Balmer lines used in the metallicity calibrations. $g$-band fibre covering fractions are required to be at least 20 per cent, enforcing an effective lower redshift cut $z \sim 0.04$. An upper redshift cut of $z<0.1$ is also imposed. Galaxies dominated by active galactic nucleus (AGN) emission are removed using the criteria presented in Kewley et al. (2006). The resulting sample contains 28,000 star-forming galaxies. Emission line fluxes were corrected by measuring the Balmer decrement and applying the Milky Way extinction curve of Cardelli et al. (1989).

Kewley \& Ellison (2008) considered 10 different metallicity calibrations: the direct (or $T_{e}$ ) method and nine different strong line methods. $T_{e}$ based metallicities are not available for the majority of individual SDSS galaxies, hence we consider it no further here (but see (Andrews \& Martini 2013, Curti et al. 2017) for works that have derived $T_{e}$ based metallicities for SDSS spectral stacks). Of the nine strong line methods, we exclude two from our current work. First, we exclude the calibration of Denicoló et al. (2002); this calibration is based on the ratio of $[\mathrm{NII}] / \mathrm{H} \alpha$ and has been superseded by several other calibrations used in this work. Second, we also exclude Pilyugin \& Thuan 2005); as shown by Kewley $\&$ Ellison (2008) this diagnostic does not cross-calibrate well with other methods.

Below we briefly review the remaining seven strong line methods used by Kewley \& Ellison (2008) that we will re-calibrate in the work presented here:

- McGaugh (1991). Hereafter M91, this calibration uses $R_{23}$ and is calibrated using theoretical models. Kewley \& Ellison (2008) calibrate the upper and lower branches of this diagnostic with 
separate polynomial fits, whose degeneracy is broken with the $[\mathrm{NII}] /[\mathrm{OII}]$ ratio.

- Zaritsky et al.(1994). Hereafter Z94, this is an average of three previously published theoretically calibrated $R_{23}$ diagnostics and is applicable only to the upper branch.

- Kewley \& Dopita (2002). Hereafter KD02, this diagnostic is theoretically calibrated. KD02 is applied in two regimes - at high metallicities the calibration is based on the ratio [NII]/[OII], and at low metallicities it uses an average of several $R_{23}$ methods.

- Kobulnicky \& Kewley (2004). Hereafter KK04, this calibration uses the same theoretical grids as KD02. Application of the KK04 calibration is a multi-step process that entails an assessment of whether the galaxy is on the upper or lower $R_{23}$ branch, following an initial estimate of the ionization parameter from the ratio of $[\mathrm{OIII}] /[\mathrm{OII}]$. The final metallicity and ionization parameter is determined through an iterative process based on $R_{23}$.

- Pettini \& Pagel (2004) N2 and O3N2. Hereafter PP04 N2 and PP04 O3N2, these diagnostics are both empirically ${ }^{2}$ calibrated against direct $\left(T_{e}\right)$ metallicities, and use either the ratio of $[\mathrm{NII}] / \mathrm{H} \alpha(\mathrm{PP} 04 \mathrm{~N} 2)$ or additionally including $[\mathrm{OIII}] / \mathrm{H} \alpha(\mathrm{PP} 04$ O3N2). Specifically, the following indices are defined:

$N 2=\log [([\mathrm{NII}] \lambda 6583 / \mathrm{H} \alpha)]$

and

$$
O 3 N 2=\log [([\mathrm{OIII}] \lambda 5007 / \mathrm{H} \beta) /([\mathrm{NII}] \lambda 6583 / \mathrm{H} \alpha)]
$$

The advantage of both of $\mathrm{N} 2$ and $\mathrm{O} 3 \mathrm{~N} 2$ calibrations is that they are single valued and do not require dust correction of the emission line fluxes, because the lines used in the ratios are close in wavelength. However, PP04 N2 (as with any N2 based calibration) has the limitation that it tends to saturate at higher metallicities.

- Tremonti et al. (2004). Hereafter T04, this calibration uses a broader suite of emission lines, including [NII] and [SII] in addition to the commonly used oxygen and Balmer lines. Unlike the other calibrations used by Kewley \& Ellison (2008), T04 derive metallicities from a statistical assessment of a large suite of spectral synthesis models. The T04 metallicities are therefore not computed by us (nor were they by Kewley \& Ellison 2008) from the raw emission line fluxes, but rather are taken directly from the MPA/JHU cata$\log$. We note that the large number of galaxies with available T04 metallicities, despite the large number of lines required, is because of the specific $\mathrm{S} / \mathrm{N}$ requirements adopted in that work (which are not applied to all of the listed lines).

\subsection{New calibrations considered in this paper}

We include five new metallicity calibrations in the current study that were not available to Kewley \& Ellison (2008), but have become widely used in the literature since their publication. We review here their main properties, for comparison with the diagnostics used in Kewley \& Ellison (2008), but refer the reader to the original papers for full details.

- Marino et al. (2013) N2 and O3N2. Hereafter M13 N2 and M13 O3N2, this study compiled over $600 \mathrm{HII}$ regions with $T_{e}$ based metallicities. Two strong line diagnostics were calibrated

\footnotetext{
2 A small number (6/137) of the HII regions used in the Pettini \& Pagel (2004) diagnostic are calibrated using theoretical models. Given the vast majority of $T_{e}$ based metallicities used by PP04, we refer to this as an empirical method.
}

based upon this large sample, $[\mathrm{NII}] / \mathrm{H} \alpha$ (i.e. the $\mathrm{N} 2$ index) and $([\mathrm{OIII}] / \mathrm{H} \beta) /([\mathrm{NII}] / \mathrm{H} \alpha)$ (i.e. the $\mathrm{O} 3 \mathrm{~N} 2$ index). As with other $\mathrm{N} 2$ diagnostics, saturation is a problem at higher metallicities, whereas O3N2 can be used up to $12+\log (\mathrm{O} / \mathrm{H}) \sim 8.9$.

- Dopita et al. (2016). Hereafter D16, this diagnostic uses a unique set of emission lines amongst those studied here. By combining [NII], $\mathrm{H} \alpha$ and [SII], which are all located close together in wavelength space, this metallicity diagnostic offers several advantages. First, it is independent of extinction. Second, all of the emission lines can be covered in one spectral setting, even if the instrumental coverage is relatively narrow. Finally, this diagnostic is characterised by a linear function up to super-solar metallicities $(12+\log (\mathrm{O} / \mathrm{H}) \approx 9.05)$ and is largely independent of ionization parameter.

- Curti et al. (2017) N2 and O3N2. Hereafter C17 N2 and C17 O3N2, this study used over 110,000 star-forming galaxies drawn from the SDSS DR7 (the same dataset from which we will draw our sample) to derive an empirical metallicity calibration over an unprecedentedly broad range in $\mathrm{O} / \mathrm{H}$ by stacking galaxies in narrow bins of $[\mathrm{OII}]$ and $[\mathrm{OIII}]$ relative to $\mathrm{H} \beta$. The very high $\mathrm{S} / \mathrm{N}$ ratios achieved in the stacking process enable a detection of [OIII] $\lambda$ 4363 , which is required to determine the electron temperature and thereby solve for metallicity. As with Marino et al. (2013), Curti et al. (2017) calibrated their $T_{e}$ based metallicities for two strong line ratios, $\mathrm{N} 2$ and $\mathrm{O} 3 \mathrm{~N} 2$.

Table 1 summarizes the 12 diagnostics used in this paper; seven from the original Kewley \& Ellison (2008) work and five new ones. Table 1 also summarizes the metallicity regime over which the calibrations are valid and the emission lines used.

\subsection{SDSS DR7 Galaxy Selection}

Emission line fluxes used in this work are taken from the publicly available SDSS DR7 MPA/JHU catalo 3 e.g. Brinchmann et al. (2004). The emission lines in this catalog have been corrected for underlying stellar absorption and for Galactic extinction. We further correct for internal extinction by assuming an intrinsic $\mathrm{H} \alpha / \mathrm{H} \beta=2.85$ and a Small Magellanic Cloud extinction curve (Pei $1992 \sqrt{4}$

We begin by selecting all galaxies from the DR7 that are classified as star-forming according to the Kauffmann et al. (2003) definition. For this selection, we require that the $\mathrm{S} / \mathrm{N}$ in the four emission lines required for the star-forming classification have a $\mathrm{S} / \mathrm{N}>$ 3. Approximately 159,000 galaxies are thus selected.

In order to identify galaxies for which robust, global metallicities may be estimated, further cuts are required on emission line $\mathrm{S} / \mathrm{N}$ and fibre covering fraction. In order to facilitate an equitable comparison with the conversions of Kewley \& Ellison (2008), we adopt the same criteria as in that work, i.e., $g$-band fibre covering fraction of at least 20 per cent, an upper redshift cut of 0.1 and a minimum $\mathrm{S} / \mathrm{N}$ in the emission lines required for a given calibration of 8. The application of the first two of these criteria result in a sample that is reduced from $\sim 159,000$ to $\sim 61,000$. The $\mathrm{S} / \mathrm{N}$ requirement further reduces the sample to a size that depends on the specific set of emission lines required for a given calibration. The final column of Table 1 summarizes the number of galaxies selected for each calibration. The number of galaxies used in each pairwise

\footnotetext{
3 https://wwwmpa.mpa-garching.mpg.de/SDSS/

4 We test the influence of choosing a Milky Way type extinction curve and find that it has negligible impact on the calculated metallicities.
} 
Table 1. The list of metallicity calibrations used in this paper and number of galaxies in the DR7 dataset.

\begin{tabular}{lcccc}
\hline Metallicity & Reference & Range of validity & Lines required & $N$ galaxies \\
\hline M91 & McGaugh (1991) & $7.24<12+\log (\mathrm{O} / \mathrm{H})<9.4$ & {$[\mathrm{NII}] \lambda 6583,[\mathrm{OII}] \lambda 3727,[\mathrm{OIII}] \lambda 5007, \mathrm{H} \beta$} & 32275 \\
Z94 & Zaritsky et al. (1994) & $12+\log (\mathrm{O} / \mathrm{H})>8.35$ & {$[\mathrm{NII}] \lambda 6583,[\mathrm{OII}] \lambda 3727,[\mathrm{OIII}] \lambda 5007, \mathrm{H} \beta$} & 31701 \\
KD02 & Kewley \& Dopita (2002) & $8.2<12+\log (\mathrm{O} / \mathrm{H})<9.4$ & {$[\mathrm{NII}] \lambda 6583,[\mathrm{OII}] \lambda 3727,[\mathrm{OIII}] \lambda 5007, \mathrm{H} \beta$} & 37899 \\
KK04 & Kobulnicky \& Kewley (2004) & $7.6<12+\log (\mathrm{O} / \mathrm{H})<9.2$ & {$[\mathrm{NII}] \lambda 6583,[\mathrm{OII}] \lambda 3727,[\mathrm{OIII}] \lambda 5007, \mathrm{H} \beta$} & 32168 \\
PP04 N2 & Pettini \& Pagel (2004) & $-2.5<\mathrm{N} 2<-0.3$ & {$[\mathrm{NII}] \lambda 6583, \mathrm{H} \alpha$} & 44726 \\
PP04 O3N2 & Pettini \& Pagel (2004) & $\mathrm{O} 3 \mathrm{~N} 2<2.0$ & {$[\mathrm{OIII}] \lambda 5007, \mathrm{H} \beta, \mathrm{H} \alpha,[\mathrm{NII}] \lambda 6583$} & 44590 \\
T04 & Tremonti et al. (2004) & $\mathrm{N} / \mathrm{A}$ & {$[\mathrm{NII}] \lambda 6583,[\mathrm{OII}] \lambda 3727,[\mathrm{OIII}] \lambda 5007,[\mathrm{OI}]$} & 50774 \\
& & & $\mathrm{HeI}, \mathrm{H} \beta, \mathrm{H} \alpha,[\mathrm{SII}] \lambda \lambda 6717,6731$ & 45036 \\
M13 N2 & Marino et al. (2013) & $-1.6<\mathrm{N} 2<-0.2$ & {$[\mathrm{NII}] \lambda 6583, \mathrm{H} \alpha$} & 44085 \\
M13 O3N2 & Marino et al. (2013) & $-1.1<\mathrm{O} 3 \mathrm{~N} 2<1.7$ & {$[\mathrm{OIII}] \lambda 5007, \mathrm{H} \beta, \mathrm{H} \alpha,[\mathrm{NII}] \lambda 6583$} & 42892 \\
D16 & Dopita et al. (2016) & $7.76<12+\log (\mathrm{O} / \mathrm{H})<9.05$ & {$[\mathrm{NII}] \lambda 6583, \mathrm{H} \alpha,[\mathrm{SII}] \lambda \lambda 6717,6731$} & 43743 \\
C17 N2 & Curti et al. (2016) & $7.6<12+\log (\mathrm{O} / \mathrm{H})<8.85$ & {$[\mathrm{NII}] \lambda 6583, \mathrm{H} \alpha$} & 44946 \\
C17 O3N2 & Curti et al. (2016) & $7.6<12+\log (\mathrm{O} / \mathrm{H})<8.85$ & {$[\mathrm{OIII}] \lambda 5007, \mathrm{H} \beta, \mathrm{H} \alpha,[\mathrm{NII}] \lambda 6583$} & 449 \\
\hline
\end{tabular}
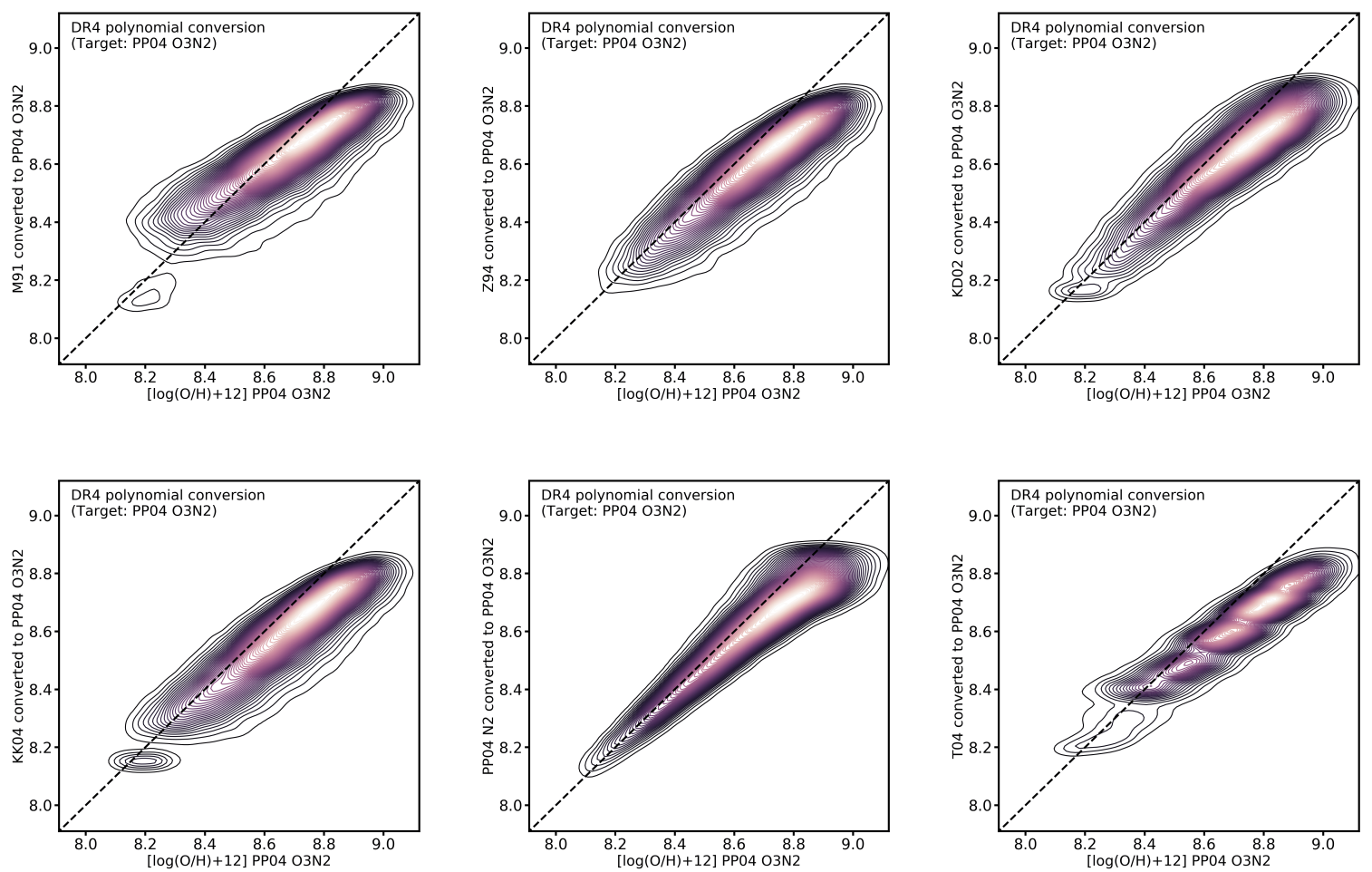

Figure 1. Assessment of the DR4-derived metallicity conversions on the expanded DR7 dataset. In each panel, the DR7-derived PP04 O3N2 metallicity is shown on the x-axis. For the six other strong line methods calibrations with conversions by Kewley \& Ellison (2008) that are used in this paper, the y-axis shows each metallicity diagnostic converted to PP04 O3N2 using the original DR4 polynomial coefficients. The deviations of the data from the diagonal 1:1 dashed line demonstrate that the DR4 conversions are not optimized for the DR7 dataset.

cross-calibration (which requires a $\mathrm{S} / \mathrm{N}>8$ in the lines used for both calibrations in a given conversion) is further given in Tables A1-A12

\section{THE POLYNOMIAL CONVERSION METHOD}

Derived from a sample of $\sim 28,000$ star-forming galaxies from the SDSS DR4, Kewley \& Ellison (2008) presented tabulated coefficients for a third order polynomial that could be used to convert between different metallicity calibrations. We begin by re-assessing the validity of these metallicity conversions as applied to our larger DR7 dataset. We will then extend these polynomial based conversions to include the five additional diagnostics summarized in Section 2.2

\subsection{A re-assessment of Kewley \& Ellison (2008) conversions using SDSS DR7 data}

Of the ten original metallicity diagnostics explored in Kewley \& Ellison (2008), conversions between eight of the strong line metallicity calibrations were presented in Kewley \& Ellison (2008). Of 

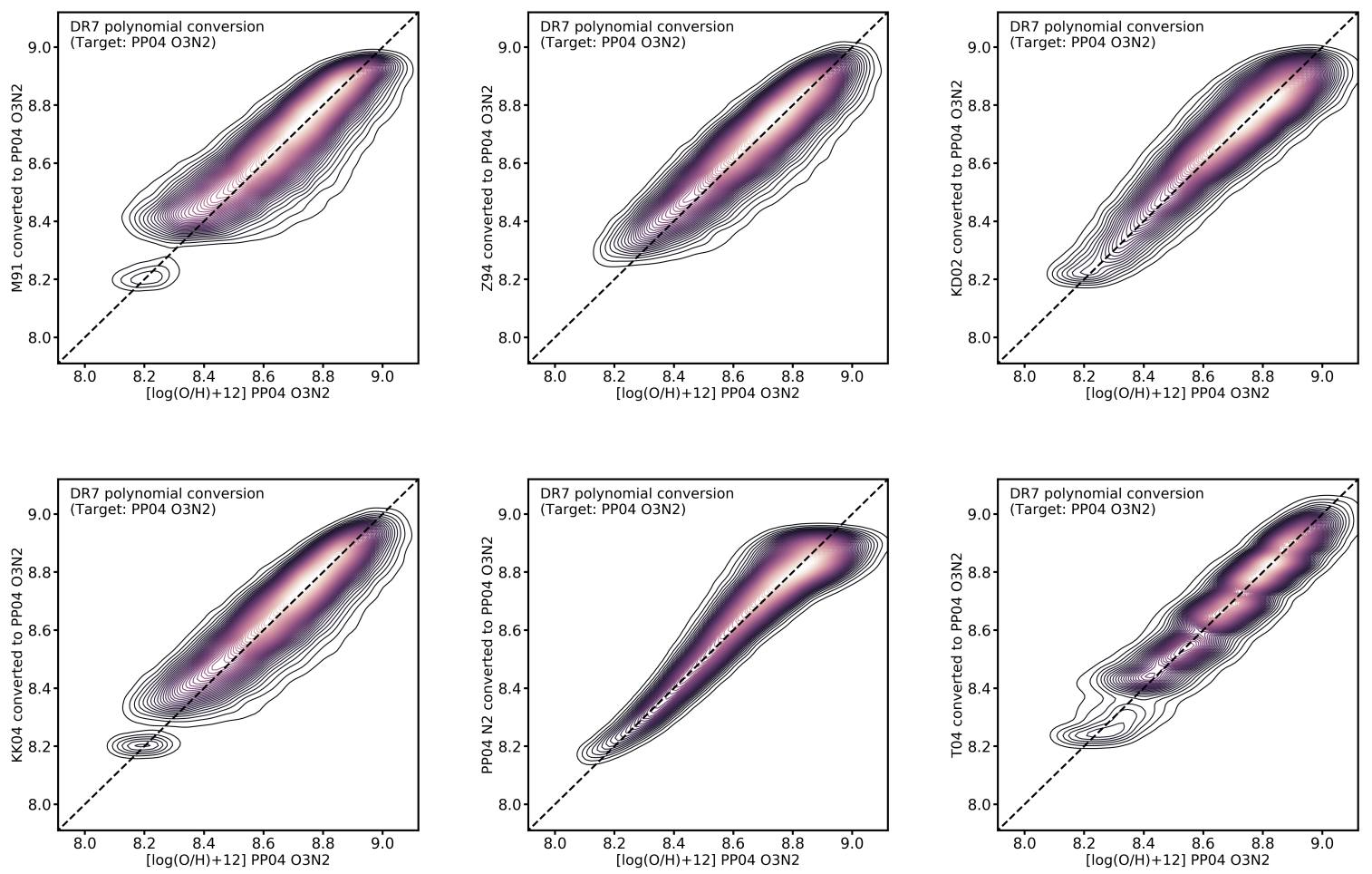

Figure 2. The same plot as Fid 1 but with the newly determined polynomial coefficients derived from the DR7 dataset. The systematic offsets found in Fid1 using the DR4 coefficients have now been removed and the converted metallicities perform well.

these eight, we have removed Denicolo et al. (2002), for the reasons described in Sec. 2.1 leaving the following seven strong line diagnostics: M91, Z94, KD02, KK04, PP04 N2, PP04 O3N2 and T04. Gas phase metallicities are computed for the first six of these diagnostics (the T04 metallicity is taken directly from the MPA/JHU catalog) for our DR7 sample of galaxies (see the last column of Table 1 for the number of galaxies available for each diagnostic).

Fig. 11 shows some examples of the Kewley \& Ellison (2008) conversion coefficients (derived for DR4) as applied to our DR7 dataset. For this exemplar, we have selected the PP04 O3N2 as the target diagnostic, which is shown on the $\mathrm{x}$-axis of each panel of Fig. 1 for the DR7 dataset. Each of the other six diagnostics is then converted onto the PP04 O3N2 system using the polynomial coefficients derived by Kewley \& Ellison (2008). If the conversions worked perfectly, then the converted version of a given calibration (y-axis) should be identical to its directly measured value (x-axis), and the points will line up along the diagonal 1:1 dashed line.

The results shown in Fig. 1 (and a thorough assessment of all the combinations of targets and conversions, not shown here for brevity) confirm that the Kewley \& Ellison (2008) DR4-derived coefficients are not optimized for the DR7 dataset. The offsets between the converted and target metallicities are typically quite small, $<0.1$ dex, but the large dataset shows that these effects are statistically significant, and depending on the calibration in question, can affect a significant fraction of galaxies.

We determine that the origin of these offsets and biases is likely due to changes in the emission line flux values, which are typically higher in the DR7 than in the DR4. While uniform enhancements in the line fluxes should not produce systematic biases in metallicity values, we find that not all emission lines are stronger in the DR7 by the same percentage, which will present a bias when comparing metallicities based on different emission line ratios. Fig. 1 shows conversions between calibrations which use different emission line ratios, and are therefore strongly affected by this change in line strengths. Conversions between calibrations based on the same emission lines are less discrepant between the two data sets.

We therefore re-compute improved coefficients between the seven metallicity diagnostics in common between our sample and that of Kewley \& Ellison (2008), using an identical functional form of a third order polynomial. The new coefficients are tabulated in Tables A1-A7 Fig. 2 shows the same combination of conversions as Fig 1 but using these updated polynomial coefficients. The systematic offsets and biases that were present in Fig 1 have now been largely eliminated.

\subsection{Polynomial coefficients for converting five additional diagnostics}

In addition to the diagnostics previously presented in Kewley \& Ellison (2008), we have additionally derived conversions, using the polynomial method, for the five additional diagnostics summarized in $\mathrm{Sec} 2.2$ (M13 N2, M13 O3N2, D16, C17 N2 and C17 O3N2). We again use a third-order polynomial, in order to be consistent with the other diagnostic conversions. The coefficients for these new diagnostic conversions are presented in Tables A8- A12

In Fig. 3 we show an example of these new conversions, using C17 O3N2 as the target metallicity diagnostic. Similar to Figs 1 and 2 . the $\mathrm{C} 17 \mathrm{O} 3 \mathrm{~N} 2$ metallicities (derived directly from the SDSS DR7 spectra) in a given calibration are plotted on the x-axis, with each of the other diagnostics, converted into $\mathrm{C} 17 \mathrm{O} 3 \mathrm{~N} 2$ via their poly- 

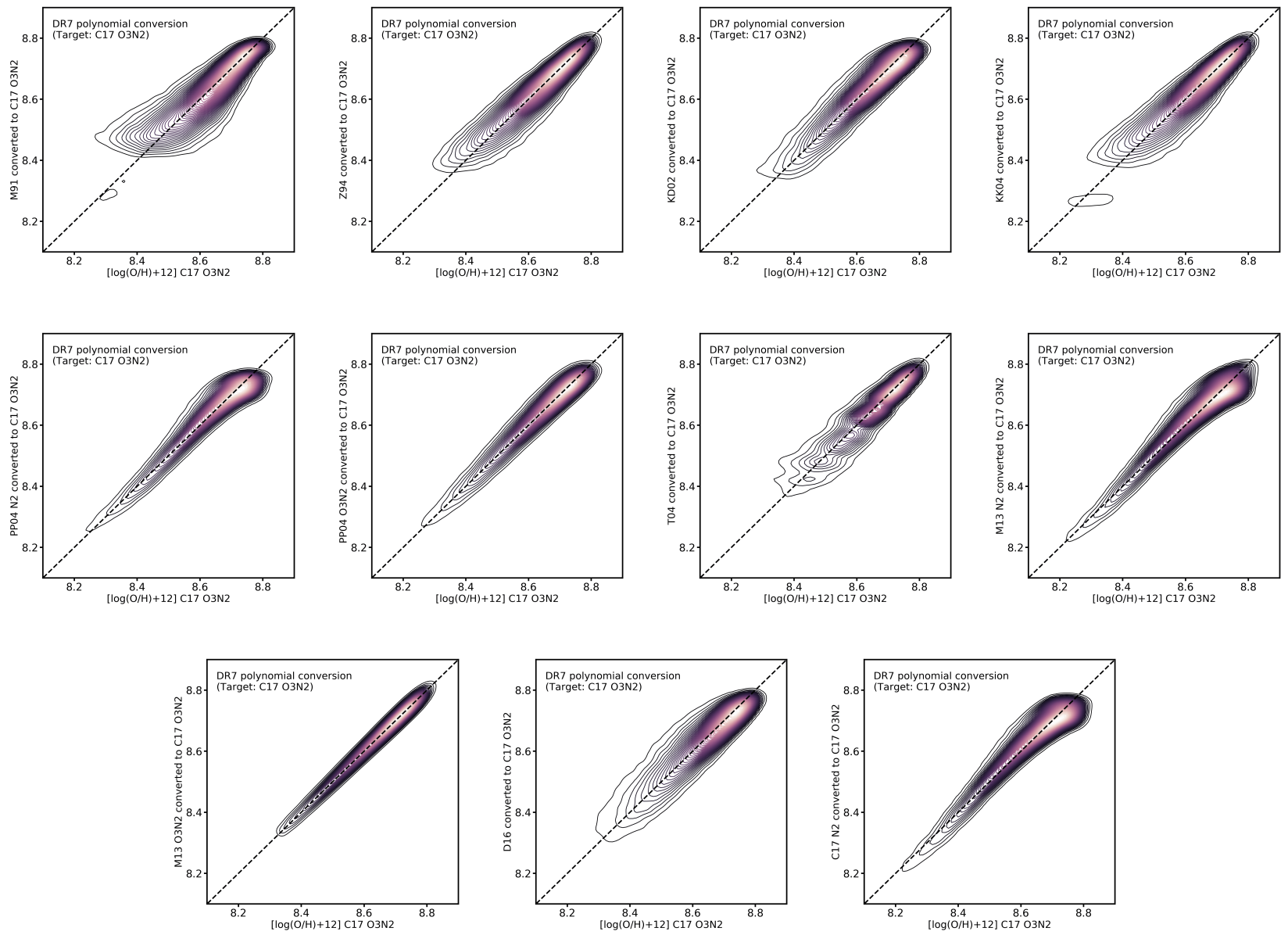

Figure 3. Metallicity conversions using the polynomial method and DR7 data for an expanded set of diagnostics. In this example, C17 O3N2 is the target metallicity diagnostic and the other 11 calibrations have been converted to it using the coefficients in the Appendix tables.

nomial fit, are plotted on the y-axis. The new conversions show no systematic offsets or skewed behaviour, confirming that the thirdorder polynomial fit between pairs of calibrations is a good representation of the data.

Taken together, the updated coefficients in Tables A1- A12 represent an improvement in both accuracy and available diagnostics over the original work on this topic by Kewley \& Ellison (2008). Given the cumbersome nature of the table format, we have also developed a Graphical User Interface (GUI) into which these coefficients are coded, to facilitate the use of the polynomial functions within the community. We note for transparency that the metallicities output by this GUI are rounded to three decimal places. The GUI can be downloaded here: Polynomial-link

\section{THE RANDOM FOREST METHOD}

Figures 2 and 3 show that, for the 12 strong line metallicity calibrations presented here, a third-order polynomial can be used to convert between diagnostics with no systematic offsets. None the less, there are several reasons to motivate an investigation of non-linear solutions to these conversions. First, since a polynomial function is a linear model based on coefficients, it is less able to capture any nonlinear features in the correlations between calibrations than a random forest model which is nonlinear by construction. A further potential advantage of a machine learning approach is that degeneracy breaking decisions (such as upper and lower branch of $R_{23}$ ) are naturally included in the model (see also the discussion in Ucci et al. 2018; Ho et al. 2019).

\subsection{Methodology}

A random forest (RF) is a supervised and non-parametric model used for regression and classification problems. In a non-parametric model, there is no internal parameter to train and learn. Since an RF is a supervised method, it implies that for a vector of input parameters, there is a target vector that we want to predict. An RF consists of several similar decision trees - the building blocks of the random forest model. A decision tree is a system of yes/no options (see Fig 4 for a schematic of the process). This system is a recursive partitioning model in which, from the root node, the input data is repeatedly divided and sub-divided. First, the root node is split into two (internal) nodes based on the binary decisions on an input value of the data under study. Then the procedure is repeated for the two nodes (i.e., in layer-2). In this way, the depth can be increased. The depth of a tree is a parameter that is chosen by the user. We can stop the tree from growing according to some criteria such as the maximum depth or the minimum samples in a node. The end result is a decision tree with final nodes that are called leaves. Each 


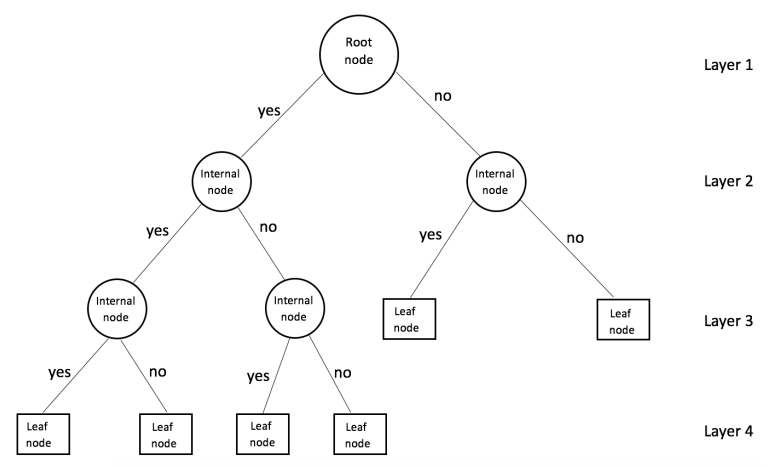

Figure 4. A schematic diagram of the structure of a decision tree. A random forest is comprised of many individual decision trees, which mitigates the problem of over-fitting the data.

leaf consists of a set of arranged input parameters and the corresponding target value. In a regression problem, the average value of the targets in a leaf will be predicted in that leaf. A single tree has the potential for overfitting as we increase the depth of the tree. To tackle this problem, we can extend the use of a single decision tree to a 'forest' (Tin Kam Ho 1998).

In a RF model, a set (e.g., 100) of similar trees are made (i.e., with the same depth and the same decision procedure). Input to the first tree is a portion (e.g., 20\%) of the primary input data, which is randomly selected (with replacement) and then fed to the tree. This procedure is repeated for all trees one by one. In this way, each tree's prediction will be a slightly different from other trees due to the randomization procedure. The final regression prediction is the average of all predicted results of all similar trees. This method prevents overfitting and generally gives better and more accurate results. One advantage of using an RF model is that for a two-branch problem, such as $R_{23}$ metallicity conversions in Kewley \& Ellison (2008), one single RF model can predict the metallicity values in branches, without the need for a user-defined break point between the branches. An RF approach therefore not only reduces the number of required models, but also removes the need for subjective boundaries. In this work, we use the RF package from scikit-learn (Pedregosa et al. 2011). We use an RF with 100 estimators (trees) and increase the depth of the trees to have at least 10 members (metallicities) in leaves, which is found to be the optimal number for the present work.

\subsection{Results from the random forest}

Fig. 5 has the same format as Fig. 3, i.e. using $\mathrm{C} 17 \mathrm{O} 3 \mathrm{~N} 2$ as the target and converting from the 11 other strong line methods. Fig. 5 shows that the RF models have successfully converted from the base to the target metallicity diagnostic with no systematic offsets or skewness.

Visually, Figs. 5 (RF conversions with $\mathrm{C} 17 \mathrm{O} 3 \mathrm{~N} 2$ as the target) and 2 (DR7 polynomial fits with $\mathrm{C} 17 \mathrm{O} 3 \mathrm{~N} 2$ as the target) look very similar. In Fig. 6 we compare the two methods quantitatively by plotting the scatter within our new polynomial (blue circles) and random forest (red squares) conversions. In this example, C17 O3N2 is again the target metallicity and the scatter is shown for each of the other 11 metallicity calibrations used in the conversion. From Fig. 6, it can be seen that for any given base calibration, when converting to the $\mathrm{C} 17 \mathrm{O} 3 \mathrm{~N} 2$ calibration, the scatter for the two techniques is almost identical. We have found this to be generally true for any combination of metallicity diagnostics, indicating that in terms of overall performance, the polynomial and RF methods are equally strong. Therefore, the random forest approach does not offer any performance advantage over the polynomial method ${ }^{5}$ However, operationally, the random forest is simpler for the user, requiring no subjective assessment of degeneracies or iterative solution for ionization parameter. We caution that this RF model has been trained and tested on the DR7 data set only, and may not transfer well to other data sets.

In order to facilitate the application of our RF model within the community, we have developed a GUI for all metallicity conversions in this paper. The interface uses 132 different RF models for the conversions. As with the polynomial GUI, the metallicities output by this RF GUI are rounded to 3 decimal places. The code can be downloaded from (RF-link) and uses the same format as the polynomial GUI also provided as a companion to this paper.

Kewley \& Ellison (2008) demonstrated (their Fig. 2) that the mass-metallicity relation shows a range of 0.7 dex in metallicity (at fixed stellar mass) for the suite of calibrations used in that paper. Kewley \& Ellison (2008) also demonstrated (their Fig. 4) that after the application of their metallicity conversions, these variations were effectively removed. In Fig. 7 and Fig. 8 we repeat this demonstration with the full suite of 12 calibrations used in this paper and the application of the random forest conversions. In Fig. 77. we show a third order polynomial fit to the mass-metallicity relation for the 12 calibrations in the original metallicity sample, prior to any conversions, described in Table 1 The stellar masses come from the MPA-JHU data release of Brinchmann et al. (2004). Broadly consistent with the results of Kewley \& Ellison (2008), we find a 0.6 dex range in metallicity between calibrations at fixed stellar mass. This is slightly less than the range found by Kewley \& Ellison (2008) because we have excluded the most deviant calibration (Pilyugin \& Thuan 2005) from our suite. In Fig. 8 we show the improvement to the mass-metallicity relation for the DR7 sample after the application of the RF metallicity conversions. In each of the 12 panels, one of the metallicity calibrations is selected in turn as the target. The other 11 diagnostics are then converted to this target using our RF model. For each of the 12 metallicity calibrations, it can be seen that after the RF conversion has been applied, the mass-metallicity relation is invariant to the choice of metallicity diagnostic.

\section{SUMMARY}

We have presented a three step re-assessment of the metallicity calibration conversions originally presented in Kewley \& Ellison (2008). First, we have approximately doubled the number of starforming galaxies in the sample, by extending the original DR4 sample used by Kewley \& Ellison (2008) to the DR7. Our complete star-forming galaxy sample, with cuts on covering fraction and redshift, contains $\sim 61,000$ galaxies and the samples for the individual metallicity calibrations (which have further cuts on emission line $\mathrm{S} / \mathrm{N}$ ) typically contain $\sim 40,000$ galaxies. Seven of the strong line metallicity calibrations presented in Kewley \& Ellison (2008) are considered in this work. We find that the metallicity conversions derived by Kewley \& Ellison (2008) are not optimized for use on the DR7 dataset (Fig. 1). The second stage of our analysis

\footnotetext{
5 Because of the discrete nature of the RF 'leaf', there may be more clustering around particular target metallicities in the RF model, compared to the polynomial conversion. The preservation of equal magnitudes of scatter indicates that this is not causing major biases in the conversion process.
} 

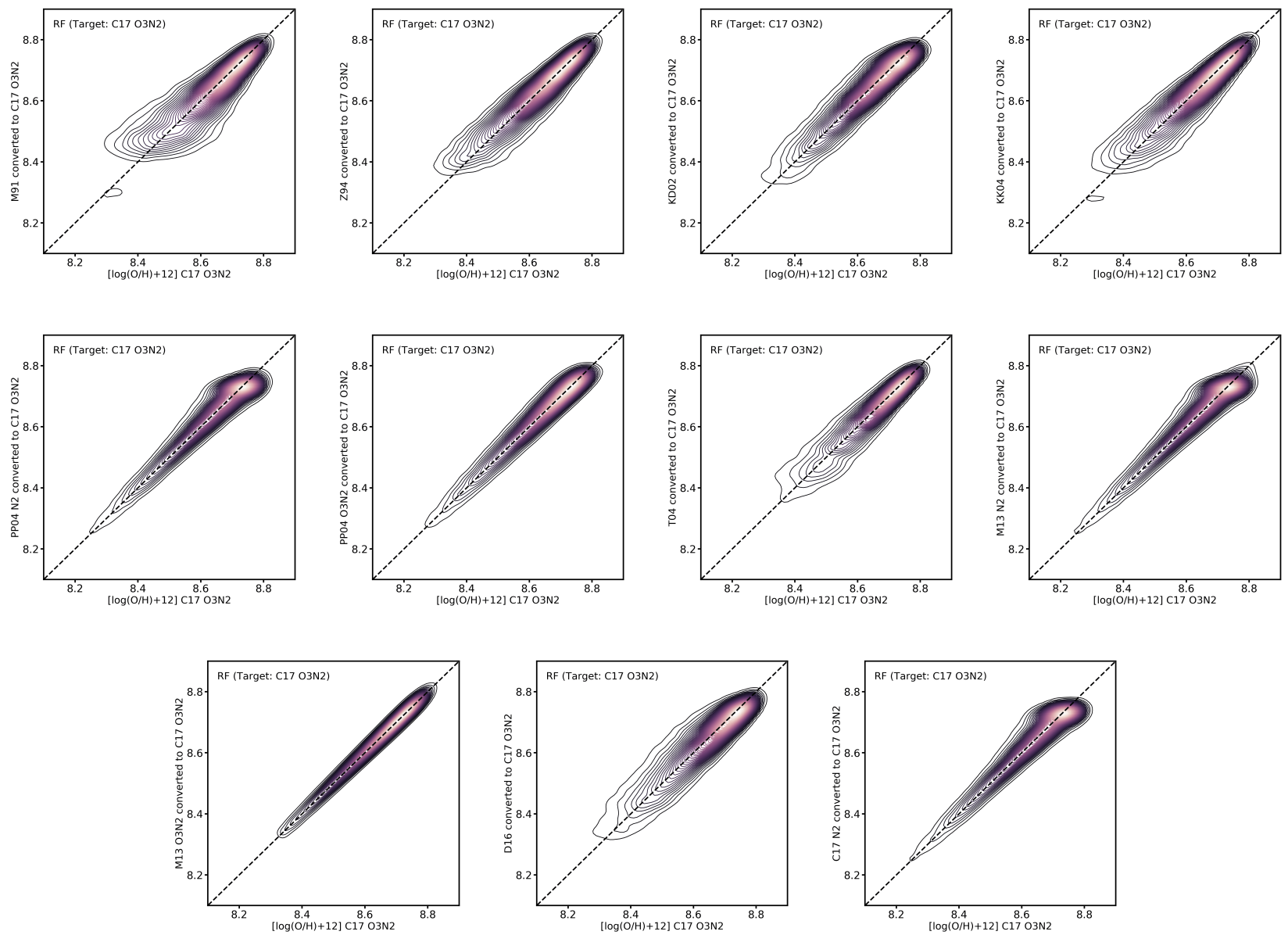

Figure 5. The same as Fig. 3 but using a random forest method for calculating the metallicity conversions.

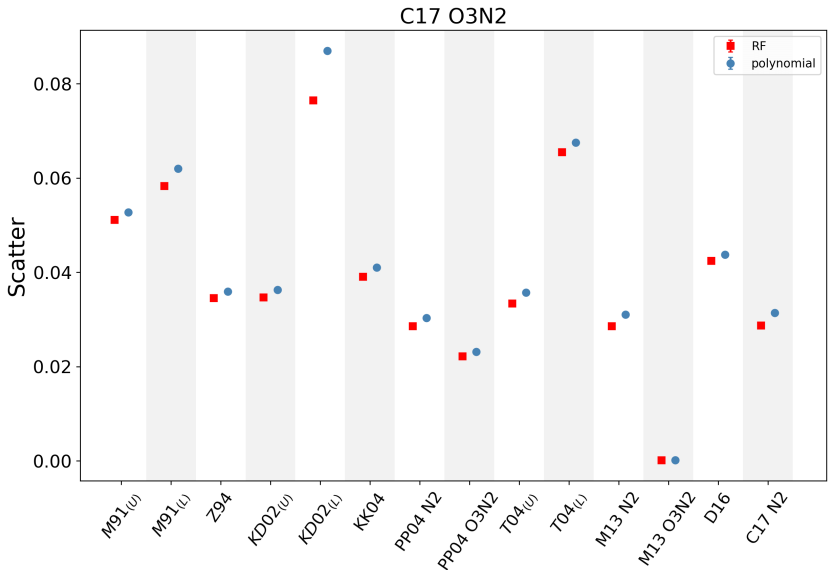

Figure 6. A comparison between the scatter obtained by the new polynomial equations and the RF results. The results do not show a significant difference. The title shows the base, and on the ' $x$ ' axis the input is shown.

is therefore to repeat the third order polynomial fitting procedure used by Kewley \& Ellison (2008) to derive new coefficients (Tables A1- A7) which better represent the DR7 data (Fig. 2). We also derive coefficients for five additional strong line metallicity diagnostics that have been presented in the literature since the original

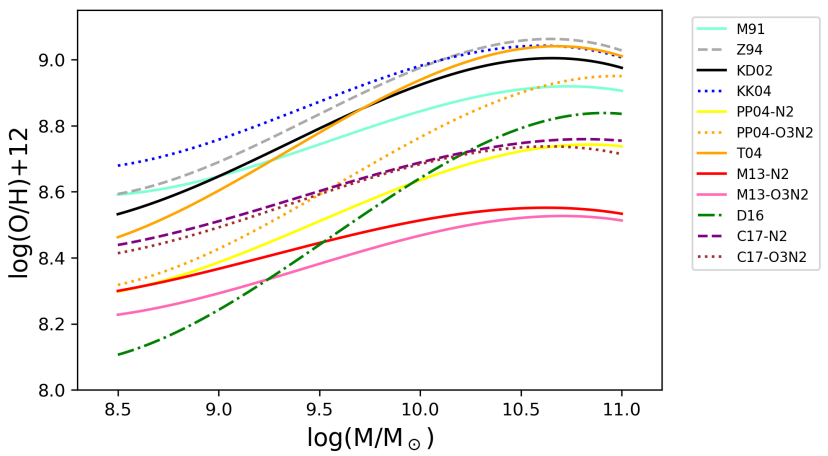

Figure 7. Third order polynomial fits to the mass-metallicity relations for the original metallicity sample as recalculated for the DR7, for each of the 12 calibrations, described in Table 1

work of Kewley \& Ellison (2008) (Fig. 3 and Tables A8- A12. Taken together, the coefficients presented in Table A1-A12 represent the most complete polynomial based conversion factors for global galaxy metallicities.

The third stage of our re-assessment of metallicity conversions focuses on the potential improvement over polynomial fits that machine learning methods may provide. Such methods can potentially better capture complexity in the data, and have the specific advan- 


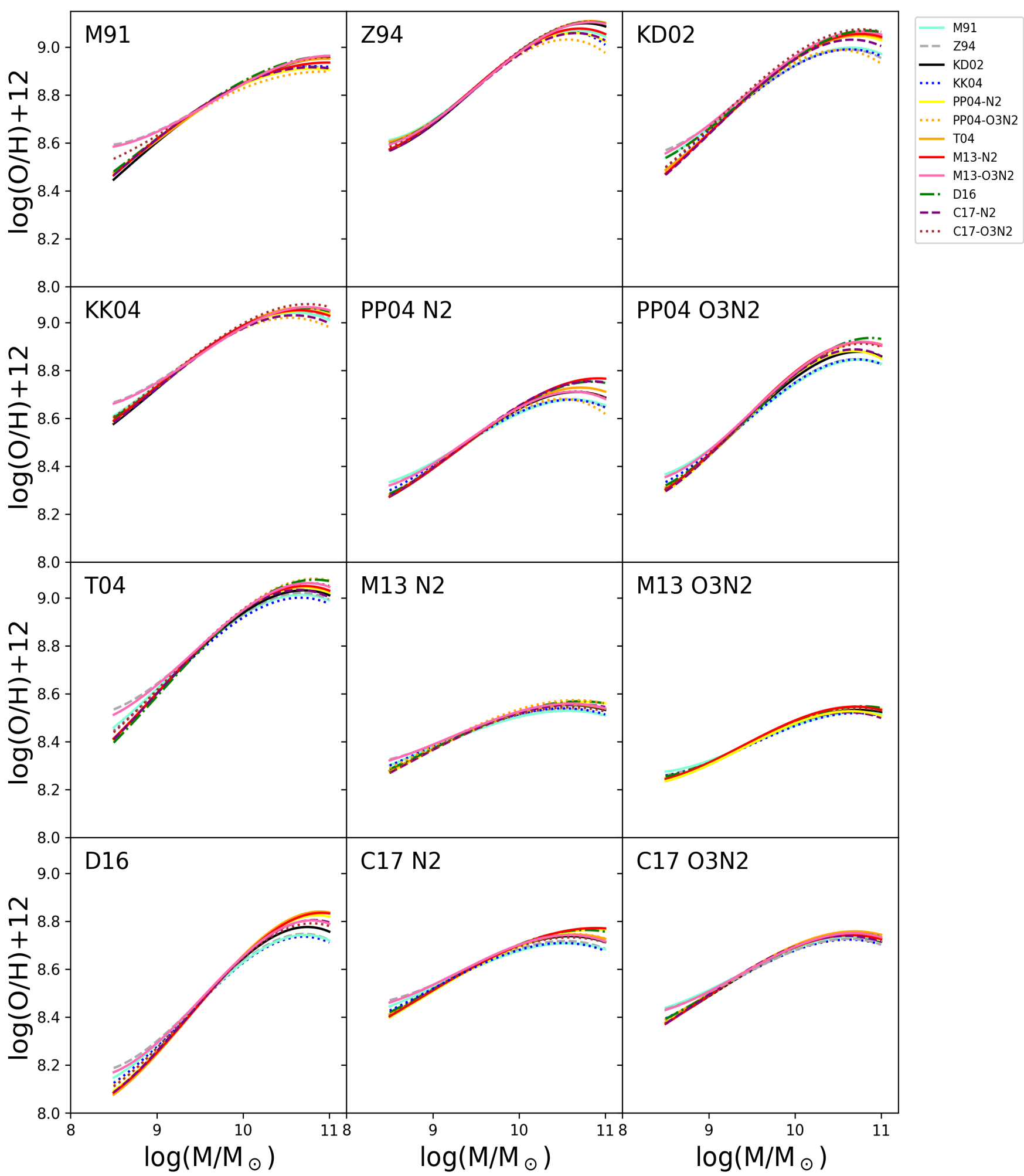

Figure 8. The mass-metallicity relations derived from the DR7 data after application of the random forest conversions. In each panel, one of the 12 metallicity calibrations is selected as the target (top left legend) and the other 11 diagnostics are converted to this target. The curves are all in excellent agreement, showing that the conversions have successfully re-calibrated each diagnostic to a common scale. 
tage in the realm of metallicity determinations of not requiring separate functions for upper and lower branch $R_{23}$ calibrations. We successfully model the metallicity conversions between each of the 12 diagnostics presented in this work (Fig 5). Despite the additional potential of the random forest compared to a simple third order polynomial fit, the two methods perform very similarly. The predicted metallicity conversions are very similar (e.g. comparing Fig. 3 and Fig. 5 and the uncertainties of the two methods are almost identical (Fig. 6). Nonetheless, the RF offers the afore-mentioned advantage of avoiding uncertainty in decisions concerning upper and lower branch $R_{23}$ branches, and therefore may be more robust for metallicities in the turnover regime at intermediate metallicities. We have made our random forest models publicly available in a user-friendly format for use by the community.

\section{ACKNOWLEDGEMENTS}

SLE gratefully acknowledges the receipt of an NSERC Discovery Grant.

We are grateful to the MPA/JHU groups for making their SDSS catalogs public. The work presented here would not have been possible without this resource.

Funding for the SDSS and SDSS-II has been provided by the Alfred P. Sloan Foundation, the Participating Institutions, the National Science Foundation, the U.S. Department of Energy, the National Aeronautics and Space Administration, the Japanese Monbukagakusho, the Max Planck Society, and the Higher Education Funding Council for England. The SDSS Web Site is http://www.sdss.org/

The SDSS is managed by the Astrophysical Research Consortium for the Participating Institutions. The Participating Institutions are the American Museum of Natural History, Astrophysical Institute Potsdam, University of Basel, University of Cambridge, Case Western Reserve University, University of Chicago, Drexel University, Fermilab, the Institute for Advanced Study, the Japan Participation Group, Johns Hopkins University, the Joint Institute for Nuclear Astrophysics, the Kavli Institute for Particle Astrophysics and Cosmology, the Korean Scientist Group, the Chinese Academy of Sciences (LAMOST), Los Alamos National Laboratory, the Max-Planck-Institute for Astronomy (MPIA), the MaxPlanck-Institute for Astrophysics (MPA), New Mexico State University, Ohio State University, University of Pittsburgh, University of Portsmouth, Princeton University, the United States Naval Observatory, and the University of Washington.

\section{DATA AVAILABILITY}

We have developed two GUIs for all polynomial and RF metallicity conversions in this paper. Each interface uses 132 different set of coefficients and models for the conversions. The data underlying this article are available in:

Polynomial:(Polynomial-link)

Random Forest: (RF-link)

\section{References}

Acquaviva V., Raichoor A., Gawiser E., 2015, ApJ, 804, 1, 8 Andrews B. H., Martini P., 2013, ApJ, 765, 2, 140

Bluck A. F. L., Bottrell C., Teimoorinia H., et al., 2019, MNRAS, $485,1,666$
Bottrell C., Hani M. H., Teimoorinia H., et al., 2019, MNRAS, 490, 4, 5390

Brinchmann J., Charlot S., White S. D. M., et al., 2004, MNRAS, 351, 4, 1151

Cardelli J. A., Clayton G. C., Mathis J. S., 1989, ApJ, 345, 245

Ćiprijanović A., Snyder G. F., Nord B., Peek J. E. G., 2020, Astronomy and Computing, 32, 100390

Curti M., Cresci G., Mannucci F., Marconi A., Maiolino R., Esposito S., 2017, MNRAS, 465, 2, 1384

Denicoló G., Terlevich R., Terlevich E., 2002, in Revista Mexicana de Astronomia y Astrofisica Conference Series, edited by W. J. Henney, J. Franco, M. Martos, vol. 12 of Revista Mexicana de Astronomia y Astrofisica Conference Series, 257-257

Dey B., Rosolowsky E., Cao Y., et al., 2019, MNRAS, 488, 2, 1926

Dopita M. A., Kewley L. J., Sutherland R. S., Nicholls D. C., 2016, Ap\&SS, 361, 61

Ellison S. L., Teimoorinia H., Rosario D. J., Mendel J. T., 2016, MNRAS, 455, 1, 370

Ellison S. L., Thorp M. D., Lin L., et al., 2020, MNRAS, 493, 1, L39

Ferreira L., Conselice C. J., Duncan K., Cheng T.-Y., Griffiths A., Whitney A., 2020, ApJ, 895, 2, 115

Ho I. T., 2019, MNRAS, 485, 3, 3569

Huertas-Company M., Rodriguez-Gomez V., Nelson D., et al., 2019, MNRAS, 489, 2, 1859

Kauffmann G., Heckman T. M., Tremonti C., et al., 2003, MNRAS, 346, 4, 1055

Kewley L. J., Dopita M. A., 2002, ApJS, 142, 1, 35

Kewley L. J., Ellison S. L., 2008, ApJ, 681, 2, 1183

Kewley L. J., Groves B., Kauffmann G., Heckman T., 2006, MNRAS, 372, 3, 961

Kewley L. J., Nicholls D. C., Sutherland R. S., 2019, ARA\&A, 57,511

Kobulnicky H. A., Kewley L. J., 2004, ApJ, 617, 1, 240

López-Sánchez Á. R., Koribalski B. S., van Eymeren J., et al., 2012, MNRAS, 419, 2, 1051

Marino R. A., Rosales-Ortega F. F., Sánchez S. F., et al., 2013, A\&A, 559, A114

McGaugh S. S., 1991, ApJ, 380, 140

Osterbrock D. E., 1989, Astrophysics of gaseous nebulae and active galactic nuclei

Pagel B. E. J., 1989, Rev. Mexicana Astron. Astrofis., 18, 161

Pedregosa F., Varoquaux G., Gramfort A., et al., 2011, Journal of machine learning research, 12, Oct, 2825

Pérez-Montero E., Amorín R., 2017, MNRAS, 467, 2, 1287

Pettini M., Pagel B. E. J., 2004, MNRAS, 348, 3, L59

Pilyugin L. S., Thuan T. X., 2005, ApJ, 631, 1, 231

Stasińska G., 2006, A\&A, 454, 3, L127

Teimoorinia H., Bluck A. F. L., Ellison S. L., 2016, MNRAS, 457, 2, 2086

Teimoorinia H., Ellison S. L., 2014, MNRAS, 439, 4, 3526

Teimoorinia H., Ellison S. L., Patton D. R., 2017, MNRAS, 464, 4, 3796

Teimoorinia H., Kavelaars J. J., Gwyn S. D. J., Durand D., Rolston K., Ouellette A., 2020a, AJ, 159, 4, 170

Teimoorinia H., Toyonaga R. D., Fabbro S., Bottrell C., 2020b, PASP, 132, 1010, 044501

Tin Kam Ho, 1998, IEEE Transactions on Pattern Analysis and Machine Intelligence, 20, 8, 832

Tremonti C. A., Heckman T. M., Kauffmann G., et al., 2004, ApJ, $613,2,898$ 
Ucci G., Ferrara A., Pallottini A., Gallerani S., 2018, MNRAS, 477, 2, 1484

Zaritsky D., Kennicutt Robert C. J., Huchra J. P., 1994, ApJ, 420, 87

\section{APPENDIX A: POLYNOMIAL COEFFICIENTS FROM DR7 DATA}

As shown in Fig. 1 the Kewley \& Ellison (2008) polynomial coefficients derived from DR4 data are not optimized for the DR7 dataset used herein. In Tables A1- A7 we provide updated coefficients for six of the metallicity diagnostics used in Kewley \& Ellison (2008) for the DR7 dataset, using the same polynomial form, i.e.,

$y=a+b x+c x^{2}+d x^{3}$

$\mathrm{y}$ is the target calibration (i.e. what you want to convert to), and $\mathrm{x}$ is the metallicity that we want to convert from. The coefficients $a$, $b, c$, and $d$ can be found for different targets. For example, suppose that we have an estimated metallicity (x) that has been obtained by method M91. If we want to convert it to (target) Z94, then we should use the coefficients in the first row of Table A2

Furthermore, in Tables A8-A12 we present coefficients for five more recent metallicity diagnostics included in this paper that were not available to Kewley \& Ellison (2008). 
Table A1. Metallicity calibration conversion constants for different inputs (x). Target (y) = M91

\begin{tabular}{|c|c|c|c|c|c|c|}
\hline $\mathrm{x}$ & $\mathrm{X}$-range & $N$-Galaxies & $a$ & $b$ & $c$ & $d$ \\
\hline Z94 & $8.4-9.2$ & 31443 & 86.793 & -23.7914 & 2.3092 & -0.0697 \\
\hline $\mathrm{KD} 2_{(\mathrm{U})}$ & $8.4-9.2$ & 31714 & 387.3987 & -123.9031 & 13.4001 & -0.4784 \\
\hline $\mathrm{KD} 2_{(\mathrm{L})}$ & $8.1-8.4$ & 558 & 600.32489 & -144.5812 & 8.8228 & 0 \\
\hline KK04 & $8.25-9.15$ & 32102 & -2193.9176 & 751.0972 & -85.452 & 3.2434 \\
\hline PP04 N2 $(\mathrm{U})$ & $8.2-8.8$ & 31133 & -225.7426 & 79.8716 & -9.1515 & 0.3529 \\
\hline PP04 N2 $(\mathrm{L})$ & $8.05-8.3$ & 1813 & 4.7727 & -2.4793 & 0.3550 & 0 \\
\hline $\mathrm{PP} 4 \mathrm{O} \mathrm{N} 2_{(\mathrm{U})}$ & $8.2-8.9$ & 29042 & -1033.7866 & 363.4906 & -42.3049 & 1.6435 \\
\hline $\mathrm{PP} 04 \mathrm{O} 3 \mathrm{~N} 22_{(\mathrm{L})}$ & $8.05-8.4$ & 3589 & -370.6417 & 90.1443 & -5.3562 & 0 \\
\hline $\mathrm{T} 04_{(\mathrm{U})}$ & $8.2-9.2$ & 30871 & -354.349 & 125.8661 & -14.6117 & 0.5678 \\
\hline $\mathrm{T} 4_{(\mathrm{L})}$ & $8.05-8.4$ & 1160 & 1582.9942 & -588.1495 & 72.992 & -3.0104 \\
\hline $\mathrm{M} 13 \mathrm{~N} 2_{(\mathrm{U})}$ & $8.2-8.7$ & 31890 & 98.523 & -22.6664 & 1.425 & 0 \\
\hline M13 N2 & $8.0-8.3$ & 1809 & -15.9858 & 2.9668 & 0 & 0 \\
\hline M13 O3N2 & $8.1-8.7$ & 31613 & 91.0036 & -20.7636 & 1.3061 & 0 \\
\hline $\mathrm{D} 16_{(\mathrm{U})}$ & $8.0-9.1$ & 30758 & 53.5881 & -16.7644 & 2.0224 & -0.0788 \\
\hline $\mathrm{D} 16_{(\mathrm{L})}$ & $7.8-8.0$ & 536 & -8.4116 & 2.0972 & 0 & 0 \\
\hline $\mathrm{C} 17 \mathrm{~N} 2_{(\mathrm{U})}$ & $8.3-8.9$ & 31470 & 95.6496 & -33.318 & 4.092 & -0.1619 \\
\hline $\mathrm{C} 17 \mathrm{~N} 2_{(\mathrm{L})}$ & $8.1-8.3$ & 257 & -3.2641 & 1.3817 & 0 & 0 \\
\hline C17 O3N2 & $8.25-8.9$ & 32070 & 57.9884 & -12.5818 & 0.7971 & 0 \\
\hline
\end{tabular}

Table A2. Metallicity calibration conversion constants for different inputs (x). Target $(y)=\mathbf{Z 9 4}$

\begin{tabular}{lcccccc}
\hline $\mathrm{x}$ & $\mathrm{x}$-range & $N$-Galaxies & $a$ & $b$ & $c$ & $d$ \\
\hline M91 & $8.4-9.1$ & 31909 & 1825.2332 & -627.9727 & 72.194 & -2.7599 \\
KD02 & $8.4-9.2$ & 31680 & 1012.4977 & -340.8346 & 38.446 & -1.4403 \\
KK04 & $8.55-9.2$ & 31835 & -590.5284 & 203.7411 & -23.225 & 0.8876 \\
PP04 N2 & $8.05-8.8$ & 30912 & 2619.495 & -924.9867 & 109.0873 & -4.2822 \\
PP04 O3N2 & $8.05-8.9$ & 28917 & 406.0196 & -140.3262 & 16.4181 & -0.6362 \\
T04 & $8.4-9.2$ & 29952 & 830.7654 & -280.1803 & 31.7195 & -1.1925 \\
M13 N2 & $8.1-8.7$ & 31701 & 145.2596 & -34.2738 & 2.1454 & 0 \\
M13 O3N2 & $8.15-8.7$ & 31433 & 1437.9466 & -507.8459 & 59.9503 & -2.3507 \\
D16 & $7.9-9.1$ & 30745 & 468.8946 & -164.737 & 19.5684 & -0.7711 \\
C17 N2 & $8.2-8.9$ & 31172 & 10.5919 & -1.9581 & 0.2038 & 0 \\
C17 O3N2 & $8.1-8.9$ & 31701 & 391.6684 & -124.3462 & 13.2068 & -0.4563 \\
\hline
\end{tabular}

Table A3. Metallicity calibration conversion constants for different inputs (x). Target (y) = KD02

\begin{tabular}{lcccccc}
\hline $\mathrm{x}$ & X-range & $N$-Galaxies & $a$ & $b$ & $c$ & $d$ \\
\hline M91 $(\mathrm{U})$ & $8.5-9.1$ & 31560 & 234.9239 & -87.0053 & 10.8854 & -0.4451 \\
M91 & $8.05-8.3$ & 382 & 69.0297 & -15.5757 & 0.9969 & 0 \\
Z94 & $8.4-9.2$ & 31445 & -82.8695 & 27.6615 & -2.8345 & 0.0993 \\
KK04 & $8.2-9.2$ & 32168 & 99.3232 & -31.9684 & 3.6341 & -0.1331 \\
PP04 N2 & $8.2-8.9$ & 37304 & 491.9975 & -178.472 & 21.7866 & -0.8797 \\
PP04 N2 & $8.05-8.3$ & 1839 & 335.5263 & -81.3035 & 5.0493 & 0 \\
PP04 O3N2 & $8.2-8.9$ & 33017 & -353.9603 & 121.8415 & -13.7148 & 0.5177 \\
PP04 O3N2 & $8.05-8.3$ & 1420 & 129.8919 & -30.8517 & 1.9554 & 0 \\
T04 & $8.2-9.2$ & 36029 & 289.3069 & -97.0787 & 11.0951 & -0.4188 \\
T04 & $8.05-8.4$ & 1186 & -139.3008 & 50.8218 & -5.9044 & 0.232 \\
M13 N2 & $8.2-8.7$ & 37497 & 2113.0985 & -744.0017 & 87.4392 & -3.4156 \\
M13 N2 & $8.0-8.2$ & 377 & 450.6873 & -109.3972 & 6.7626 & 0 \\
M13 O3N2 & $8.15-8.7$ & 37214 & -1831.2202 & 651.7518 & -77.1251 & 3.04923 \\
D16 & $8.0-9.1$ & 35930 & -105.37 & 36.6551 & -3.962 & 0.1447 \\
C17 N2 & $8.3-8.9$ & 36758 & -39.973 & 9.7639 & -0.4758 & 0 \\
C17 N2 & $8.1-8.3$ & 258 & 29.1097 & -5.3562 & 0.3433 & 0 \\
C17 O3N2 & $8.25-8.9$ & 31992 & -770.9174 & 271.901 & -31.7625 & 1.2428 \\
C17 O3N2 & $8.0-8.25$ & 174 & -61.3755 & 16.8597 & -1.0199 & 0 \\
\hline
\end{tabular}


Table A4. Metallicity calibration conversion constants for different inputs (x). Target (y) = KK04

\begin{tabular}{|c|c|c|c|c|c|c|}
\hline $\mathrm{x}$ & $\mathrm{x}$-range & $N$-Galaxies & $a$ & $b$ & $c$ & $d$ \\
\hline M91 $(\mathrm{U})$ & $8.4-9.1$ & 31919 & 1396.2622 & -483.698 & 56.06155 & -2.1602 \\
\hline $\mathrm{M} 1_{(\mathrm{L})}$ & $8.0-8.25$ & 425 & 721.01897 & -260.4697 & 31.681 & -1.28229 \\
\hline Z94 & $8.4-9.3$ & 31894 & 204.83638 & -69.615722 & 8.13631 & -0.31321 \\
\hline $\mathrm{KD} 02_{(\mathrm{U})}$ & $8.4-9.2$ & 31689 & 1146.076 & -388.5092 & 44.1343 & -1.6671 \\
\hline $\mathrm{KD} 02_{(\mathrm{L})}$ & $8.05-8.3$ & 356 & 428.7191 & -153.4709 & 18.5653 & -0.74416 \\
\hline PP04 N2 $2_{(U)}$ & $8.2-8.9$ & 31715 & 1137.2633 & -404.9428 & 48.3076 & -1.9159 \\
\hline $\mathrm{PP} 04 \quad \mathrm{~N} 2_{(\mathrm{L})}$ & $8.05-8.3$ & 1718 & 221.1257 & -53.9172 & 3.4127 & 0 \\
\hline $\mathrm{PP} 04 \mathrm{O} 3 \mathrm{~N} 2_{(\mathrm{U})}$ & $8.2-8.9$ & 28959 & -172.8182 & 60.3876 & -6.7529 & 0.2543 \\
\hline $\mathrm{PP} 04 \mathrm{O} 3 \mathrm{~N} 2_{(\mathrm{L})}$ & $8.05-8.3$ & 1343 & 37.2557 & -8.5021 & 0.6094 & 0 \\
\hline $\mathrm{T} 04_{(\mathrm{U})}$ & $8.3-9.2$ & 30407 & 889.9372 & -301.6969 & 34.3416 & -1.2994 \\
\hline $\mathrm{T} 04_{(\mathrm{L})}$ & $8.05-8.4$ & 1071 & 3967.1747 & -1449.9755 & 176.8893 & -7.1874 \\
\hline $\mathrm{M} 13 \mathrm{~N} 2_{(\mathrm{U})}$ & $8.2-8.7$ & 31813 & 3853.3363 & -1367.5488 & 161.9577 & -6.3857 \\
\hline M13 N2(L) & $8.0-8.2$ & 345 & 610.3371 & -149.0526 & 9.2261 & 0 \\
\hline M13 O3N2 & $8.15-8.7$ & 31550 & 542.1259 & -193.5488 & 23.2532 & -0.9248 \\
\hline D16 $(\mathrm{U})$ & $8.0-9.1$ & 30695 & 259.97912 & -91.48058 & 11.02932 & -0.44022 \\
\hline $\mathrm{D} 16_{(\mathrm{L})}$ & $7.8-8.0$ & 494 & -1.86835 & 1.29895 & 0 & 0 \\
\hline $\mathrm{C} 17 \mathrm{~N} 22_{(\mathrm{U})}$ & $8.3-8.9$ & 31385 & 3705.0113 & -1298.9898 & 152.0019 & -5.9222 \\
\hline $\mathrm{C} 17 \mathrm{~N} 2{ }_{(\mathrm{L})}$ & $8.1-8.3$ & 238 & 4.148 & 0.5126 & 0 & 0 \\
\hline $\mathrm{C} 17 \mathrm{O}^{2} \mathrm{~N}_{(\mathrm{U})}$ & $8.3-8.9$ & 31749 & -374.6472 & 136.3397 & -16.2909 & 0.6538 \\
\hline $\mathrm{C} 17 \mathrm{O}^{2} \mathrm{~N} 2_{(\mathrm{L})}$ & $8.1-8.3$ & 400 & 293.2935 & -70.2175 & 4.3258 & 0 \\
\hline
\end{tabular}

Table A5. Metallicity calibration conversion constants for different inputs (x). Target (y) = PP04 N2

\begin{tabular}{lcccccc}
\hline $\mathrm{x}$ & $\mathrm{x}$-range & $N$-Galaxies & $a$ & $b$ & $c$ & $d$ \\
\hline M91 & $8.5-9.1$ & 31431 & 3613.3844 & -1238.8022 & 141.7739 & -5.4034 \\
M91 & $8.05-8.4$ & 407 & 163.9517 & -38.6531 & 2.398 & 0 \\
Z94 & $8.4-9.3$ & 31540 & 856.0385 & -290.6646 & 33.1364 & -1.2557 \\
KD02 & $8.05-9.2$ & 37572 & 865.4984 & -295.5199 & 33.8635 & -1.2895 \\
KK04 & $8.2-9.2$ & 32039 & 733.3485 & -246.8248 & 27.9109 & -1.0481 \\
PP04 O3N2 & $8.05-8.9$ & 38278 & 409.144 & -143.4804 & 17.0264 & -0.6696 \\
T04 & $8.05-9.2$ & 40978 & 524.796 & -178.1117 & 20.3914 & -0.7747 \\
M13 N2 & $8.0-8.6$ & 44686 & 163.1399 & -38.4256 & 2.3818 & 0 \\
M13 O3N2 & $8.1-8.7$ & 43759 & 3794.7691 & -1361.791 & 163.0805 & -6.5026 \\
D16 & $7.8-9.1$ & 42591 & 141.2938 & -49.2383 & 5.9996 & -0.2404 \\
C17 N2 & $8.0-8.9$ & 43741 & 1229.0742 & -424.8038 & 49.1162 & -1.8864 \\
C17 O3N2 & $8.0-8.9$ & 44611 & 1196.6207 & -416.474 & 48.5111 & -1.8776 \\
\hline
\end{tabular}

Table A6. Metallicity calibration conversion constants for different inputs (x). Target (y) = PP04 O3N2

\begin{tabular}{lcccccc}
\hline $\mathrm{x}$ & $\mathrm{x}$-range & $N$-Galaxies & $a$ & $b$ & $c$ & $d$ \\
\hline M91 (U) & $8.5-9.1$ & 31555 & 2855.1815 & -976.6748 & 111.5376 & -4.2396 \\
M91 & $8.05-8.4$ & 387 & -1545.1399 & 587.9352 & -74.1884 & 3.1208 \\
Z94 & $8.4-9.3$ & 31540 & 342.0709 & -114.2818 & 12.942 & -0.4843 \\
KD02 & $8.1-9.2$ & 37672 & 1005.4986 & -342.3498 & 39.0563 & -1.4802 \\
KK04 & $8.2-9.2$ & 32100 & 263.38 & -82.1518 & 8.6714 & -0.2985 \\
PP04 N2 & $8.05-8.8$ & 42677 & 1733.7386 & -614.419 & 72.766 & -2.8657 \\
T04 & $8.05-9.2$ & 40976 & 425.2876 & -141.5692 & 15.9152 & -0.5918 \\
M13 N2 & $8.1-8.7$ & 44584 & 155.631 & -37.0791 & 2.329 & 0 \\
M13 O3N2 & $8.15-8.7$ & 43840 & 197.388 & -65.8684 & 7.4331 & -0.2698 \\
D16 & $7.8-9.1$ & 42611 & 237.6059 & -83.634 & 10.0731 & -0.4003 \\
C17 N2 & $8.1-8.9$ & 43289 & 5361.8221 & -1875.2013 & 218.7172 & -8.4942 \\
C17 O3N2 & $8.1-8.9$ & 44586 & -185.4252 & 76.7659 & -10.1843 & 0.4513 \\
\hline
\end{tabular}


Table A7. Metallicity calibration conversion constants for different inputs (x). Target $(y)=$ T04

\begin{tabular}{lcccccc}
\hline $\mathrm{x}$ & X-range & N-Galaxies & $a$ & $b$ & $c$ & $d$ \\
\hline M91 & $8.4-9.2$ & 30651 & 2998.9561 & -1030.2075 & 118.139 & -4.5092 \\
M91 & $8.0-8.4$ & 500 & 2.0925 & 0.7576 & 0 & 0 \\
Z94 & $8.4-9.3$ & 30155 & 549.9891 & -185.833 & 21.1524 & -0.7982 \\
KD02 $_{(\mathrm{U})}$ & $8.4-9.3$ & 35852 & 521.5475 & -179.6248 & 20.834 & -0.8003 \\
KD02 $_{(\mathrm{L})}$ & $8.1-8.4$ & 559 & -2.4905 & 1.29317 & 0 & 0 \\
KK04 $_{(\mathrm{U})}$ & $8.45-9.2$ & 30654 & 1306.7925 & -437.9528 & 49.1096 & -1.8301 \\
KK04 & $8.2-8.4$ & 457 & -6.0523 & 1.7088 & 0 & 0 \\
PP04 N2 & $8.4-8.9$ & 35781 & -104.9897 & 25.3186 & -1.4041 & 0 \\
PP04 N2 & $8.0-8.4$ & 5267 & -6.825 & 1.8446 & 0 & 0 \\
PP04 O3N2 & $8.4-9.2$ & 37478 & 522.7574 & -181.3092 & 21.2074 & -0.8225 \\
PP04 O3N2 & $8.0-8.4$ & 3600 & 947.9234 & -349.4912 & 43.1432 & -1.7678 \\
M13 N2 & $8.0-8.7$ & 40624 & -804.0024 & -279.9536 & 32.5884 & -1.2538 \\
M13 O3N2 & $8.1-8.7$ & 39970 & -1821.5398 & 651.9099 & -77.5969 & 3.0867 \\
D16 & $8.1-9.1$ & 39432 & 244.9229 & -85.9962 & 10.3362 & -0.4102 \\
D16 & $7.8-8.1$ & 1608 & 581.3903 & -188.2034 & 19.9897 & -0.6774 \\
C17 N2 & $8.2-8.9$ & 39543 & -43.2538 & 10.3962 & -0.5052 & 0 \\
C17 O3N2 & $8.2-8.9$ & 40634 & -1296.48795 & 461.04 & -54.4573 & 2.1507 \\
\hline
\end{tabular}

Table A8. Metallicity calibration conversion constants for different inputs (x). Target $(y)=$ M13 N2

\begin{tabular}{lcccccc}
\hline $\mathrm{x}$ & $\mathrm{x}-$ range & $N$-Galaxies & $a$ & $b$ & $c$ & $d$ \\
\hline M91 & $8.5-9.1$ & 31560 & 1559.59 & -537.0831 & 61.9093 & -2.3757 \\
M91 & $7.9-8.4$ & 562 & 48.3787 & -10.4483 & 0.6772 & 0 \\
Z94 & $8.4-9.3$ & 31669 & 226.7235 & -77.9489 & 9.2069 & -0.3599 \\
KD02 & $8.4-9.3$ & 37326 & 380.7872 & -131.0384 & 15.2996 & -0.5928 \\
KD02 & $8.2-8.4$ & 528 & -0.7239 & 1.6589 & -0.0704 & 0 \\
KK04 & $8.55-9.2$ & 31612 & 805.682 & -273.9803 & 31.3139 & -1.1903 \\
KK04 & $8.2-8.4$ & 447 & 4.352 & 0.4586 & 0 & 0 \\
PP04 N2 & $8.0-8.9$ & 44709 & -460.0623 & 158.8308 & -17.9864 & 0.6805 \\
PP04 O3N2 & $8.0-9.2$ & 44585 & -86.985 & 28.6534 & -2.8417 & 0.093 \\
T04 & $8.55-9.2$ & 38918 & 0.3458 & -1.8454 & 0.6829 & -0.0418 \\
T04 & $8.0-8.5$ & 2020 & 574.6886 & -212.1147 & 26.3888 & -1.0909 \\
M13 O3N2 & $8.1-8.7$ & 44085 & 120.9708 & -52.0238 & 7.5124 & -0.3468 \\
D16 & $7.8-9.1$ & 42863 & -64.0936 & 22.8361 & -2.3987 & 0.0842 \\
C17 N2 & $8.0-8.9$ & 43734 & 932.1046 & -329.5358 & 39.0721 & -1.5398 \\
C17 O3N2 & $8.0-8.9$ & 44929 & 697.0506 & -247.6338 & 29.572 & -1.1729 \\
\hline
\end{tabular}

Table A9. Metallicity calibration conversion constants for different inputs (x). Target (y) = M13 O3N2

\begin{tabular}{lcccccc}
\hline x & X-range & $N$-Galaxies & $a$ & $b$ & $c$ & $d$ \\
\hline M91 $_{(\mathrm{U})}$ & $8.5-9.1$ & 31282 & 1888.2496 & -644.077 & 73.4676 & -2.7899 \\
M91 $_{(\mathrm{L})}$ & $8.0-8.3$ & 167 & -1305.8981 & 491.485 & -61.2792 & 2.547 \\
Z94 & $8.4-9.3$ & 31417 & 341.7221 & -113.7254 & 12.8692 & -0.483 \\
KD02 $_{\text {KK04 }}$ & $8.4-9.3$ & 37043 & 967.8764 & -328.278 & 37.3634 & -1.4146 \\
KK04 $_{(\mathrm{L})}$ & $8.55-9.2$ & 31362 & 536.8013 & -176.7522 & 19.6262 & -0.7232 \\
PP04 N2 & $8.2-8.4$ & 116 & 98.1808 & -21.7183 & 1.3102 & 0 \\
PP04 O3N2 & $8.2-9.9$ & 43757 & 1204.6286 & -425.8859 & 50.4443 & -1.9879 \\
T04 & $8.5)$ & 43707 & 234.8163 & -81.3845 & 9.6777 & -0.3809 \\
T04 & $8.5-9.2$ & 38815 & 182.9739 & -61.21 & 7.0837 & -0.2707 \\
M13 N2 & $8.1-8.5$ & 1477 & 2411.7063 & -867.0572 & 104.2289 & -4.175 \\
D16 & $7.8-9.65$ & 44082 & 6083.3786 & -2158.8367 & 255.5638 & -10.0782 \\
C17 N2 & $8.3-8.9$ & 42012 & 214.9474 & -74.7581 & 8.962 & -0.3559 \\
C17 O3N2 & $8.3-8.9$ & 44083 & 0.3628 & 0.9335 & 0 & -8.4027 \\
\hline
\end{tabular}


Table A10. Metallicity calibration conversion constants for different inputs (x). Target (y) = D16

\begin{tabular}{lcccccc}
\hline $\mathrm{x}$ & $\mathrm{x}$-range & $N$-Galaxies & $a$ & $b$ & $c$ & $d$ \\
\hline M91 & $8.5-9.1$ & 30613 & 2541.5169 & -873.1917 & 100.1378 & -3.8204 \\
M91 & $8.0-8.3$ & 471 & 5.1048 & 0.3513 & 0 & 0 \\
Z94 & $8.4-9.3$ & 30224 & 3706.8072 & -237.5519 & 26.8035 & -1.0031 \\
KD02 & $8.4-9.3$ & 35949 & 1292.1462 & -439.7038 & 50.0465 & -1.8928 \\
KD02 & $8.2-8.4$ & 524 & 3.3878 & 0.5506 & 0 & 0 \\
KK04 & $8.55-9.2$ & 30663 & 875.0474 & -289.4371 & 32.0548 & -1.1768 \\
KK04 & $8.2-8.4$ & 443 & 2.8759 & 0.6069 & 0 & 0 \\
PP04 N2 & $8.1-8.9$ & 41901 & 957.6926 & -337.1474 & 39.7259 & -1.5529 \\
PP04 O3N2 & $8.0-9.2$ & 41944 & 284.8894 & -99.0825 & 11.6981 & -0.4551 \\
T04 & $8.5-9.3$ & 39034 & -252.7935 & 87.9578 & -10.0078 & 0.3848 \\
T04 & $8.0-8.5$ & 2015 & 1028.8981 & -371.8225 & 45.0596 & -1.8169 \\
M13 N2 & $8.0-8.7$ & 42210 & -1633.1334 & 615.017 & -76.9143 & 3.2096 \\
M13 O3N2 & $8.15-8.65$ & 41348 & -718.1404 & 264.3618 & -32.295 & 1.3239 \\
C17 N2 & $8.0-8.9$ & 41006 & 2375.3459 & -823.6716 & 95.2887 & -3.6646 \\
C17 O3N2 & $8.0-8.9$ & 42175 & -482.1501 & 183.9562 & -23.1037 & 0.9705 \\
\hline
\end{tabular}

Table A11. Metallicity calibration conversion constants for different inputs (x). Target (y) $=$ C17 N2

\begin{tabular}{|c|c|c|c|c|c|c|}
\hline $\mathrm{x}$ & $\mathrm{x}$-range & $N$-Galaxies & $a$ & $b$ & $c$ & $d$ \\
\hline $\mathrm{M} 1_{(\mathrm{U})}$ & $8.4-9.1$ & 31170 & 2901.7049 & -994.8047 & 113.9298 & -4.3457 \\
\hline $\mathrm{M} 1_{(\mathrm{L})}$ & $8.0-8.4$ & 502 & 2.5221 & 0.7141 & 0 & 0 \\
\hline Z94 & $8.4-9.3$ & 30636 & 383.5379 & -130.358 & 15.0295 & -0.5747 \\
\hline $\mathrm{KD} 02_{(\mathrm{U})}$ & $8.4-9.2$ & 36412 & 642.9785 & -220.0287 & 25.3594 & -0.9713 \\
\hline $\mathrm{KD} 02_{(\mathrm{L})}$ & $8.2-8.4$ & 531 & -4249.5242 & 1535.7596 & -184.7107 & 7.4079 \\
\hline${\mathrm{KK} 04_{(\mathrm{U})}}$ & $8.55-9.2$ & 31083 & 986.5064 & -334.2708 & 38.0059 & -1.4373 \\
\hline${\mathrm{KK} 04_{(\mathrm{L})}}$ & $8.2-8.4$ & 452 & 3.0422 & 0.6294 & 0 & 0 \\
\hline PP04 N2 & $8.0-8.9$ & 43044 & -1175.2954 & 413.3419 & -48.1757 & 1.8745 \\
\hline $\mathrm{PP} 04 \mathrm{O} 3 \mathrm{~N} 2$ & $8.0-9.2$ & 42596 & -7.9268 & 1.0615 & 0.3627 & -0.0305 \\
\hline $\mathrm{T} 04_{(\mathrm{U})}$ & $8.55-9.2$ & 37884 & 371.1892 & -127.1447 & 14.7861 & -0.5704 \\
\hline $\mathrm{T} 04_{(\mathrm{L})}$ & $8.0-8.5$ & 2024 & 230.5072 & -90.1699 & 11.9911 & -0.5242 \\
\hline M13 N2 & $8.0-8.6$ & 43036 & -2591.8417 & 935.0384 & -112.197 & 4.4924 \\
\hline M13 O3N2 & $8.15-8.65$ & 42099 & 1256.816 & -457.1278 & 55.6542 & -2.2528 \\
\hline D16 & $7.8-9.1$ & 40982 & -83.8363 & 30.1166 & -3.295 & 0.1213 \\
\hline $\mathrm{C} 17 \mathrm{O} 3 \mathrm{~N} 2$ & $8.0-8.9$ & 42941 & 445.5391 & -158.2197 & 18.9688 & -0.7533 \\
\hline
\end{tabular}

Table A12. Metallicity calibration conversion constants for different inputs (x). Target (y) = C17 O3N2

\begin{tabular}{lcccccc}
\hline $\mathrm{x}$ & $\mathrm{x}$-range & $N$-Galaxies & $a$ & $b$ & $c$ & $d$ \\
\hline M91 $_{(\mathrm{U})}$ & $8.5-9.1$ & 31560 & 1922.6664 & -659.1048 & 75.5519 & -2.8829 \\
M91 $_{(\mathrm{L})}$ & $8.0-8.3$ & 477 & -31.2375 & 9.0558 & -0.5155 & 0 \\
Z94 & $8.4-9.3$ & 31669 & 73.9083 & -25.0406 & 3.0904 & -0.1237 \\
KD02 & $8.4-9.2$ & 37208 & 719.4539 & -245.8567 & 28.2585 & -1.0795 \\
KD02 & $8.1-8.4$ & 559 & -100.2682 & 25.5497 & -1.5024 & 0 \\
KK04 & $8.2-9.2$ & 32168 & 302.3792 & -100.4709 & 11.3665 & -0.4254 \\
PP04 N2 & $8.05-8.9$ & 44599 & 302.2795 & -112.1483 & 14.0962 & -0.5843 \\
PP04 O3N2 & $8.05-9.2$ & 44583 & 7.1195 & -4.0341 & 0.9299 & -0.0512 \\
T04 $(\mathrm{U})$ & $8.55-9.2$ & 38918 & -86.437 & 28.1687 & -2.7891 & 0.0926 \\
T04 & $8.0-8.5$ & 2025 & 431.9057 & -164.2151 & 21.06348 & -0.8947 \\
M13 N2 & $8.0-8.65$ & 44930 & 1479.4498 & -528.8548 & 63.2182 & -2.5125 \\
M13 O3N2 & $8.1-8.7$ & 44083 & -464.0953 & 161.6573 & -18.517 & 0.7109 \\
D16 & $7.8-9.1$ & 42829 & 29.5937 & -10.6313 & 1.5776 & -0.0725 \\
C17 N2 & $8.0-8.9$ & 43636 & 1860.3708 & -657.1819 & 77.5899 & -3.0477 \\
\hline
\end{tabular}

